\title{
Evaluation of obstructed defecation syndrome (ODS) using magnetic resonance defecography (MRD)
}

\author{
Arshed Hussain Parry ${ }^{*}$ (10 and Abdul Haseeb Wani
}

\begin{abstract}
Background: Obstructed defecation syndrome is associated with varying combina s of a hy ot of ano-rectal abnormalities, and no physical examination can demonstrate these abnormalities. The sent study was aimed to evaluate the spectrum of various pelvic floor abnormalities in obstructed def 20 n synoj ome (ODS).

Results: Of the total 302 patients imaged with age range of 18-72 years $\mathrm{m}$. 7 years), 218 were females, and 84 were males. Ano-rectal junction descent was the commonest abnorma observed in 273 (90.3\%) patients followed by rectocele (232) (76.8\%), rectal intussusception (93) (30.7\% d cystocele (92) (30.4\%). Cervical descent was observed in 78 (35.7\%) of female patients. Spastic perineum was spen in 27 (8.9\%) patients.

Conclusion: MRD serves as single stop shop for demonstratino and gra ling a gamut of pelvic organ abnormalities underpinning ODS which in turn helps in choosing the b-st tro nent plan for the patient.
\end{abstract}

Keywords: Obstructed defecation syndrome, Magnetros reso nce defecography, Pelvic floor dysfunction, Spastic perineum syndrome, Rectocele

\section{Background}

Constipation constitutes a major health cono globally especially among the aging pc oulation. Te, percent of Indians above the age of 5 ( ears ar found to have constipation. In the USA, con ation leads to 2.5 million physician visits $f$ vear [1]. A uniform and consistent definition for cons, pation has been elusive, and a slew of at apts have been made to arrive at a compreherm would encompacs all th myriad symptoms and manifestations of $c$ tipation Obstructed defecation syndrome (ODS) co titutes an important subset of patients of constipacion. ODS has been defined by NICE tonal Institute for health and Clinical Excele) g colnes as inability to completely evacuate e hel fecal bolus in the presence of urge to du vate $[2,3]$. Repeated unsuccessful attempts at

* Correspondence: arshedparry@gmail.com

Department of Radiodiagnosis, Sher-i-Kashmir Institute of Medical Sciences, Srinagar, Jammu and Kashmir 190011, India defecation, sense of incomplete fecal evacuation, and excessive straining at toilet pan adversely affecting the quality of life typifies this subset of constipated patients. These patients usually resort to digital maneuvers to attain rectal evacuation [3, 4]. ODS is usually associated with varying combinations of a host of ano-rectal abnormalities, and no physical examination can demonstrate these abnormalities. Dynamic MRI imaging referred to as MRD is a single stop shop to demonstrate various pelvic floor and ano-rectal abnormalities underpinning ODS. This capability of MRD to evaluate defecation process dynamically helps in demonstration of various ano-rectal and pelvic floor abnormalities and thus allows colorectal surgeons to plan a comprehensive treatment for these patients $[4,5]$. This study was undertaken to evaluate ODS with MRD. The objective of this study was to demonstrate various pelvic floor and ano-rectal abnormalities associated with ODS.

\section{SpringerOpen}

(c) The Author(s). 2020 Open Access This article is licensed under a Creative Commons Attribution 4.0 International License, which permits use, sharing, adaptation, distribution and reproduction in any medium or format, as long as you give appropriate credit to the original author(s) and the source, provide a link to the Creative Commons licence, and indicate if changes were made. The images or other third party material in this article are included in the article's Creative Commons licence, unless indicated otherwise in a credit line to the material. If material is not included in the article's Creative Commons licence and your intended use is not permitted by statutory regulation or exceeds the permitted use, you will need to obtain permission directly from the copyright holder. To view a copy of this licence, visit http://creativecommons.org/licenses/by/4.0/. 


\section{Methods}

This was a prospective study. Patients fulfilling the clinical criteria for ODS as laid down in NICE guidelines were referred to our department for MRD by the colorectal division of surgery department. A total of 302 patients were evaluated over a period of 3 years from December 2016 to January 2020. The study was performed on 1.5 Tesla superconducting magnetic resonance imager (Magnetom Avanto, Siemens Medical System) using standard pelvic coil. All the patients were subjected to preliminary sigmoidoscopy or colonoscopy to rule out any organic cause of constipation like rectal or colonic neoplasm. Patients were thoroughly explained the procedure to ensure their cooperation. A written consent was obtained in each case. Two hundred and fifty millilitre of ultrasound jelly was instilled into the rectum using a rectal tube after putting the patient in left lateral position on the MRI table. Ultrasound jelly was chosen because of its ready availability and its high T2 contrast. Diapers were given to the patients to allow them to defecate on the MRI gantry. This ensures cleanliness of the gantry table and helps patients to save blushes and avoid unnecessary embarrassment. The imaging protocol consisted of preliminary T2 weighted axial and sagittal sequences \{repetition time (TR)/echo time (TE) $2880 \mathrm{~ms} / 89 \mathrm{~ms}$; slice thickness of $3 \mathrm{~mm}$; fierd of view $200 \mathrm{~mm}$ \} to study the anatomy. Followi th 's dynamic imaging was performed using TRUFI Nrue imaging with steady state free precession) sa once hav ing a repetition time (TR) of $45.6 \mathrm{~ms}$, echo tim TE) of $1.3 \mathrm{~ms}$, slice thickness of $3 \mathrm{~mm}$, and field of vie) 340 $\mathrm{mm}$ in sagittal plane during rest, ueeze, train, and defecation (drain out) phases. Defec on o/ drain out phase was run for a sufficient me (approximately 1 to $2 \mathrm{~min}$ ). The images were analyze d $\mathrm{c}$ Apple work station by two radiologisto sessilg 9 and 10 years of experience respective in ahdo ninal radiology. The interpreting radiogrists are blind to the clinical history of patients. $M_{-}$efecogra ny images were analyzed in mid-sagittal plane cine mode using standard sagittal anatomiral planes. Pl,oo-coccygeal line (PCL) was drawn from in ariol margin of pubis to the last coccygeal arti-lation cig. 1a). H (hiatal) line was drawn from the - feri marg of pubic symphysis to the posterior wall o, ro-rutal junction (Fig. 1b). $\mathrm{H}$ line corresponds to the $\mathrm{Ac}$ or levator hiatus. $\mathrm{M}$ line was drawn perpendicular to PCL line from the posterior end of $\mathrm{H}$ line (Fig. 1b). The PCL line defines the level of pelvic floor, and the abnormal descent of pelvic structures is diagnosed when a structure descends below PCL during straining or defecation. The ano-rectal angle is the angle measured between central axis of anal canal and posterior border of distal part of rectum. Ano-rectal angle is formed by the stretch of pubo-rectalis sling on the posterior ano-rectal junction (Fig. 1c). The position of ano-rectal junction, cervix, and bladder neck was studied in all the phases. Presence and degree of bladder, cervical, and ano-rectal junction descent below PCL were studied. Presence and degree of intussusception, rectocele, and enterocele were evaluated. Ano-rectal juction descent defined as abnormal descent of ano-rer tal viunction below pubo-coccygeal line is graded into $n, 1<3$ $\mathrm{cm})$, moderate $(3-6 \mathrm{~cm})$, and severe $(>6 \mathrm{~cm})$. Rec is defined as abnormal protrusion of thectal $v$ all beyond the expected rectal contour. It gra irto mild $(<2 \mathrm{~cm})$, moderate $(2-4 \mathrm{~cm})$, an severe $(>+\mathrm{cm})$. Abnormal caudal descent of blad $r$ and cervix below pubo-coccygeal line is also ted mild, moderate, and severe. Abnormal audal cent of various pelvic structures is graded as or the otandard classification given in Table 1. Invagin on of rectal wall into its lumen is called re $\mathrm{l}$ intussusception and is classified into mucosah $\mathrm{sc}_{\mathrm{H}}$ cuon or full thickness intussusception. When i $\mathrm{l}$ intussusception extends outside anal verg ic refe red to as rectal prolapse. Enterocele is defined as $\mathrm{Ca}$, dal displacement of small bowel loops into the rec o-vesical or recto-vaginal space. Various pelVic or abnormalities were noted down. Defecation phase of MR defecography was compared with all the er three phases of defecation (i.e., rest, strain, and squeeze) combined together.

All patients included in this research gave written informed consent to publish the data contained within this study. The datasets used and/or analyzed during the current study are available from the corresponding author on reasonable request.

\section{Results}

A total of 302 patients fulfilling the clinical criteria for ODS were studied with a mean age of 54 years (range 18-72 years). With regards to gender, 218 were females, and remaining 84 were males. Ano-rectal junction descent was commonest abnormality seen in 273 (90.3\%) patients with 132 (48 \%) showing mild descent, 71 (26\%) showing moderate descent, and remaining 70 (25.6\%) showing severe descent. During maximal strain, only 101 patients showed ano-rectal junction descent, whereas defecation phase identified another 172 (63\%) patients with ano-rectal junction descent. Anterior rectocele was seen in 232 (76.8\%) patients with mild rectocele seen in 192 patients, moderate rectocele seen in 27 patients, and severe rectocele seen in 13 patients. Anterior rectocele was seen during strain phase in 151 patients, whereas defecation phase identified another $81(34.9 \%)$ patients with rectocele taking the total to 232 (76.8\%). Cystocele was seen in $92(30.4 \%)$ patients with 71 patients showing mild cystocele, 17 showing moderate cystocele, and remaining 4 patients showing severe cystocele. Only 16 


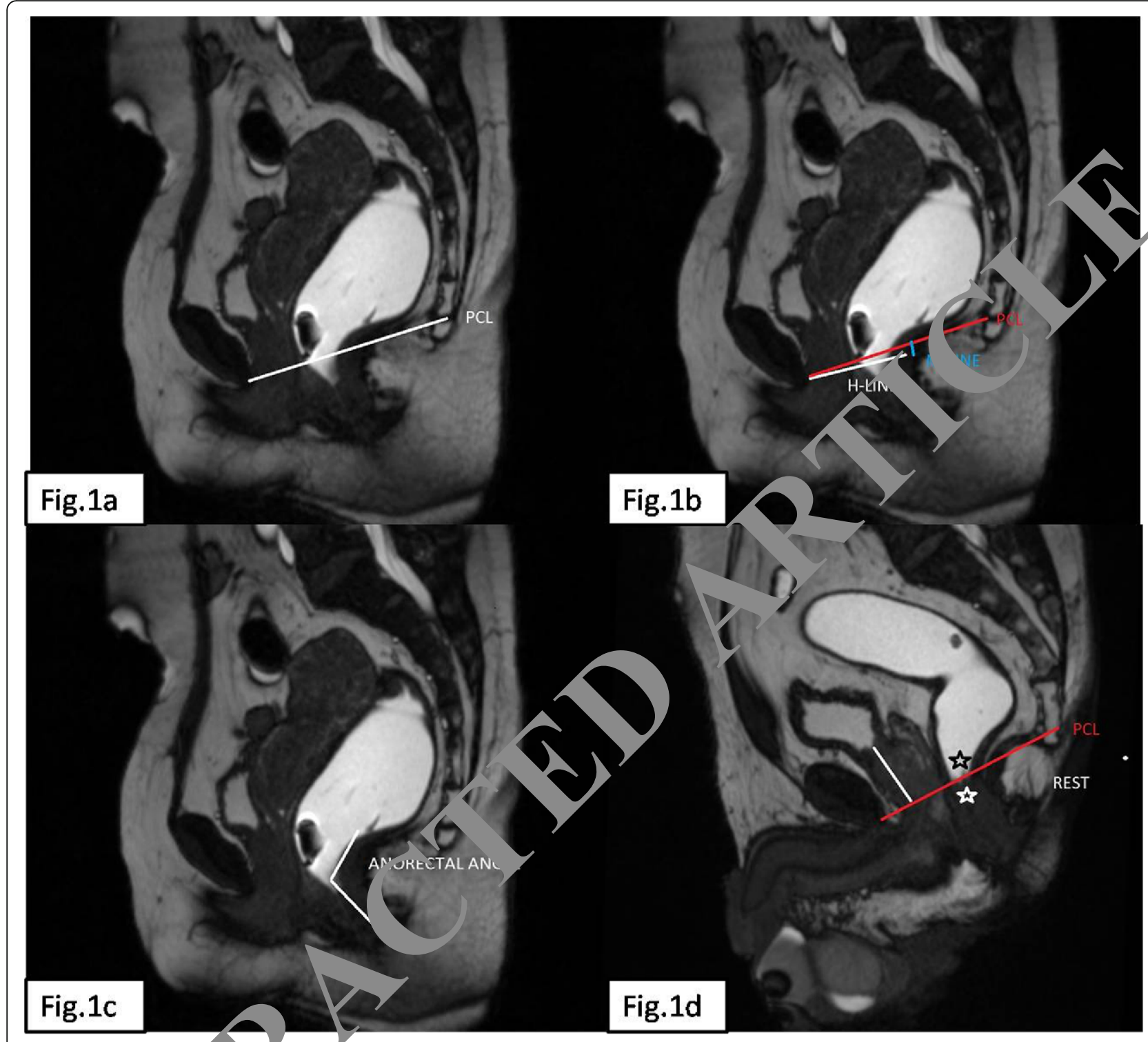

Fig. 1 Mid sagittal TRUFA in rest depicting various lines and angles for analysis of magnetic resonance defecography. Pubo-coccygeal line $(\mathrm{PCL})$ is drawn fom last veal joint to inferior margin of pubis (a) with $\mathrm{H}$ (hiatal) line drawn from inferior border of pubis to ano-rectal junction (b). The a formed b-tween long axis of anal canal and posterior rectal wall is called ano-rectal angle and is normally obtuse at rest (c). At rest, ano-rectal e and bladder neck lie above PCL (d)

$\begin{array}{lll} & & \\ & & \\ \end{array}$

patients showed some degree of bladder descent during strain phase; but during defection phase, all the $92 \mathrm{pa}-$ tients of cystocele showed bladder descent. Rectal intussusception was seen in a total of 93 (30.7\%) patients. Mucosal intussusception was seen in 69 patients, whereas 24 patients showed full thickness intussusception. Among the total study cohort, there were 4 patients of solitary rectal ulcer syndrome (SRUS) who also had presented with symptoms of outlet obstruction and thus underwent MRD. All four of them showed evidence of intussusception (3 had mucosal and 1 full thickness intussusception). Enterocele was seen in 4 patients with small bowel herniation in all the cases. Among total of 
Table 2 Various pelvic floor abnormalities observed on magnetic resonance defecography

\begin{tabular}{lllll}
\hline Abnormality & Total & Mild & Moderate & Severe \\
\hline Ano-rectal junction descent & $273(90.3 \%)$ & 132 & 71 & 70 \\
Rectocele & $232(76.8 \%)$ & 192 & 27 & 13 \\
Rectal intussusception & $93(30.7 \%)$ & & & \\
Enterocele & 4 & & & \\
Cervical descent & $78(35.7 \%)$ & 63 & 11 & 4 \\
Spastic perineum & $27(8.9 \%)$ & & & \\
Cystocele & $92(30.4 \%)$ & 71 & 17 & 4
\end{tabular}

$\$ 35.7 \%$ of female patients
218 females, cervical descent was seen in 78 (35.7\%) patients. A comparison between strain and drain out phases revealed that cervical descent was seen in only 32 (41\%) patients during maximum strain; whereas in drain out phase, all the 78 patients showed descent. Spastic perineum syndrome was seen in 27 (8.9\%) patient The entire gamut of pelvic floor abnormalities is en merated in Table 2.

\section{Discussion}

Pelvic floor dysfunction is characterizea v sladder, bowel, or sexual dysfunction with variable cymbination of pelvic organ prolapse. It affec multiparous women more commonly than men. ste. amage to pelvic

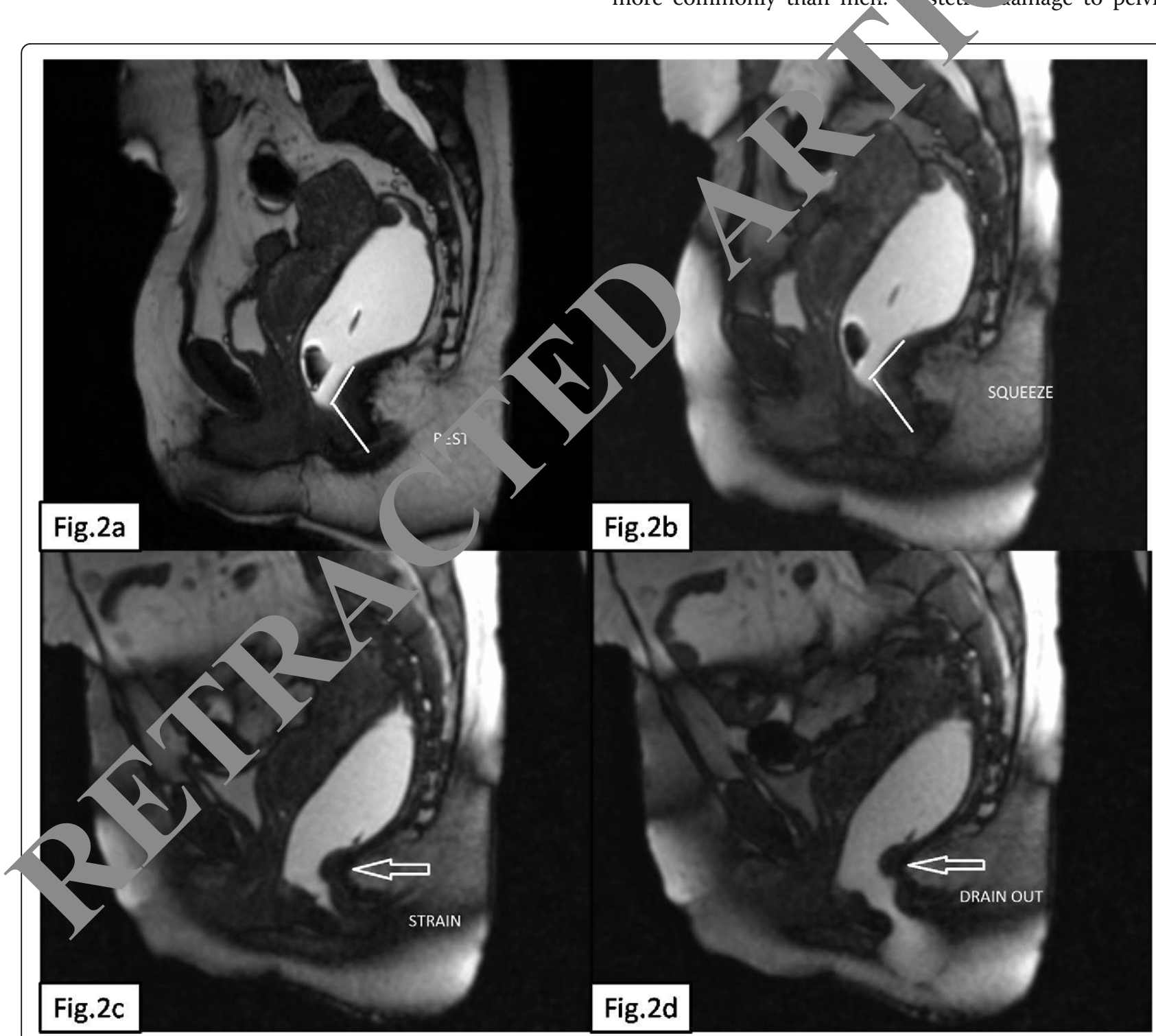

Fig. 2 Spastic perineum syndrome. Mid sagittal TRUFI images at rest (a) reveal an obtuse ano-rectal angle which decreases during squeeze (b). During the strain phase, there is further reduction in ano-rectal angle with thick pubo-coccygeal muscle (white arrow) indenting the posterior rectal wall (c).In defecation phase, there is further reduction in ano-rectal angle with prominent indentation of posterior rectal wall by the thickened pubo-rectalis muscle (d) 
floor structures like ilio-coccygeus muscle, pubococcygeus muscle, anal sphincter, endopelvic fascia, and pudendal nerve is believed to cause pelvic floor dysfunction in multiparous women. Obstructed defecation syndrome (ODS) constitutes a unique set of chronically constipated patients who fail to completely evacuate their rectum. These patients resort to excessive straining and digital maneuvering of rectum to attain complete rectal evacuation. ODS can result either from a functional abnormality or organic ano-rectal abnormality. Patients with functional abnormality can be treated with bio feedback therapy or psychotherapy, whereas those with an organic ano-rectal disorder respond to surgical correction [5]. The diagnostic armamentarium chiefly consists of fluoroscopic defecography and magnetic resonance defecography (MRD) [5-7]. MRD has the capability of demonstrating the various pelvic floor abnormalities with great accuracy. MRD serves as n one stop shop for studying the normal pelvic anat my and the complete range of pelvic floor abnormaliti $\mathrm{NRD}$ lacks radiation exposure. MRD can be performed sitting position using open configuration $M$, or in supine position using closed configuration magn [7]. MRD performed in supine position yie ds compar, ole results

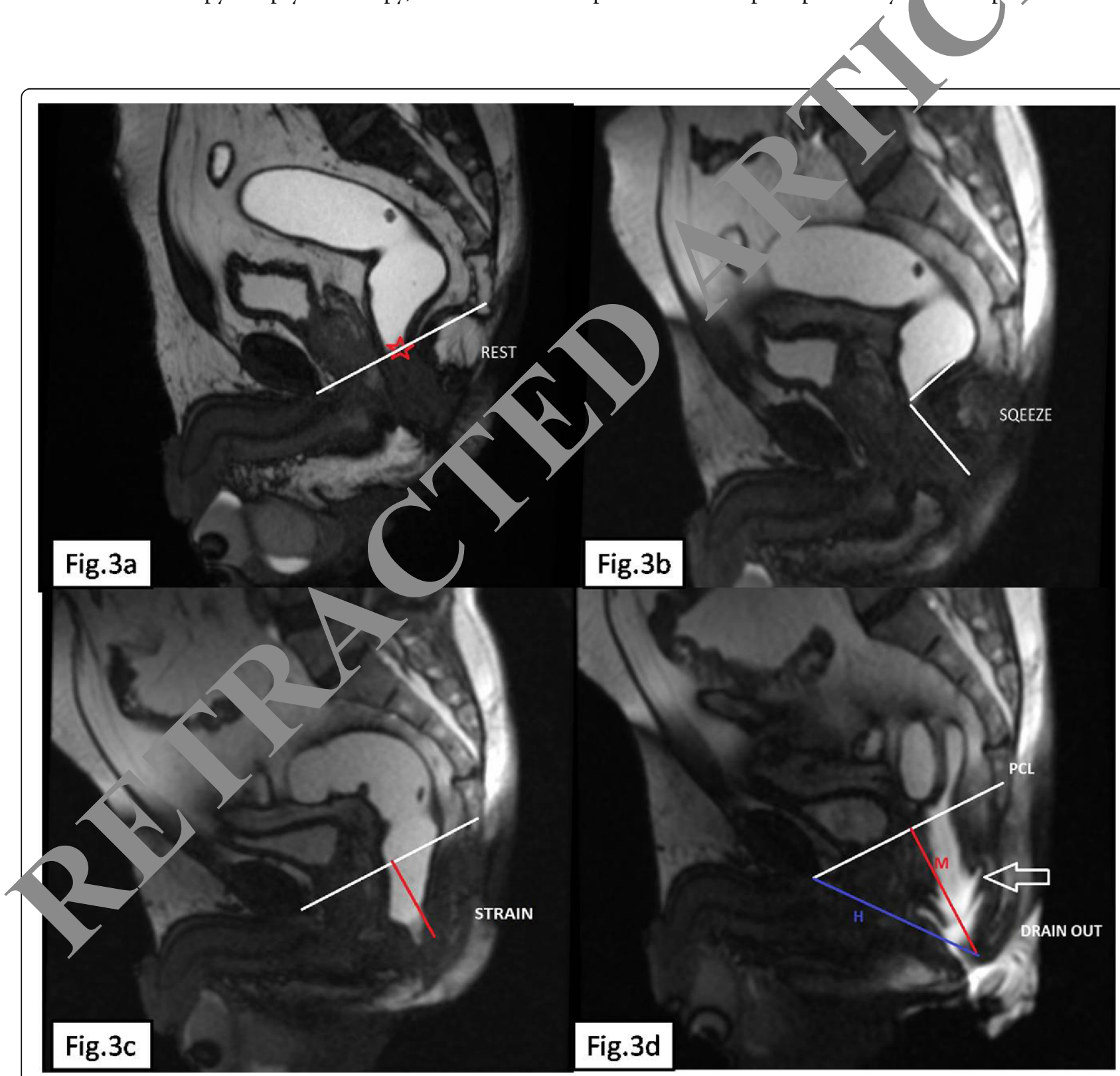

Fig. 3 Ano-rectal junction descent with rectal intussusception. During rest, ano-rectal junction (red star) is at the level of PCL (a). During squeeze (b), there is slight decrease in ano-rectal angle. During strain, ano-rectal junction (red line) shows a descent of $4.2 \mathrm{~cm}$ (c). During the defecation phase, there is further descent of ano-rectal junction (red line) with rectal intussusception (white arrow in d) 
to that performed in sitting position for the reason that the straining forces applied during defecation are of sufficient magnitude to elicit the various pathologies $[8,9]$.

Pelvic floor is divided into three compartments: anterior compartment comprises of bladder and urethra, middle compartment comprises of uterus and vagina, and the posterior compartment is comprised of ano-rectal canal $[9,10]$. However, all the three compartments work in unison, and combined disorders of pelvic floor are common and should be assessed simultaneously. Normal ano-rectal angle measures between $108^{\circ}$ and $127^{\circ}$ [11, 12]. During normal defecation, the pubo-rectalis sling relaxes leading to widening of the ano-rectal angle by $15-20^{\circ}$ so that the rectum and anal canal are aligned in a straight line to allow expulsion of fecal matter [13, 14]. Failure of widening of ano-rectal angle during defecation with persistence of acute ano-rectal angle forms the basis for the diagnosis of spastic perineum syndrome (SPS) (Fig. 2) (Video 1). This disorder is also ca"ed as paradoxical pubo-rectalis syndrome (PPS). I results from failure of pubo-rectalis muscle to rela aring defecation. In fact, there is paradoxical contract of this muscle during defecation which pre ts ope ning of ano-rectal angle during defecation with co quent failure of evacuation of feces. Thicl ening of pl,oo-rectalis muscle has been reported previou in lit rature in PPS

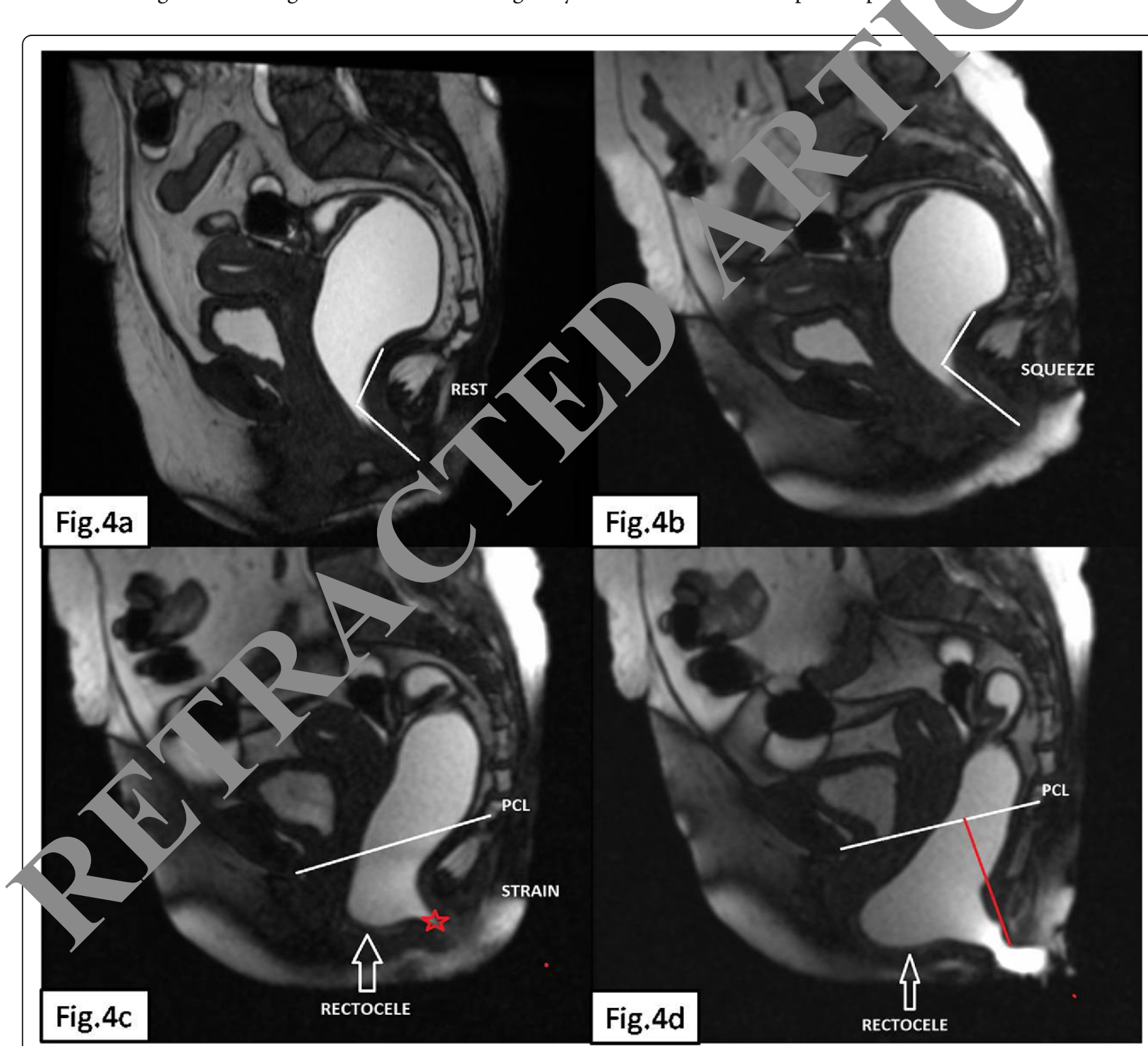

Fig. 4 Rectocele. There is reduction in ano-rectal angle from $109^{\circ}$ at rest (Fig.4a) to $92^{\circ}$ during squeeze (b). During strain (c), there is ano-rectal junction descent (red star) with formation of anterior rectocele (white arrow). In defecation phase (d), there is further descent of ano-rectal junction $(>6 \mathrm{~cm}$ ) with further enlargement of anterior rectocele $(\mathbf{d})$. This highlights the value of defecation phase which elicits or adds to various pelvic floor abnormalities 
patients [12]. However, Liu et.al in their study concluded that though mean thickness of the pubo-rectalis muscle was more in patients with PPS than in patients without PPS, but the difference between groups was not statistically significant [15]. However, they reported a significant difference in apparent diffusion co-efficient (ADC) values of pubo-rectalis muscle between patients with PPS and patients without PPS which points to the fact that alteration in muscle microstructure might be the underlying mechanism for PPS [15]. Ano-rectal junction descent was the commonest abnormality encountered with $273(90.3 \%)$ patients demonstrating various grades of ano-rectal junction descent (Fig. 3). Descent of anorectal junction can occur in isolation but frequently descent of the anterior, and middle compartment structures are also seen in association with it. This is frequently associated with feeling of incomplete evacuation resulting in further increase in straining during defecation and consequent neuropathic injury that may result in incontinence [12]. Anterior rectocele was second commonest abnormality observed in 232 (76.8\%) (Fig. 4). Factors that increase the likelihood of developing a rectocele include birth trauma, hysterectomy, chronically increased intra-abdominal pressure, and increased age. Rectoceles assume clinical relevance when symptoms develop as they are responsible for obstructed defecation w/ich usually requires vaginal or perineal digitations to ta $?$ rectal emptying [12]. Post defecation retentip $1 \mathrm{~N}$ of $\mathrm{MV}$ within rectocele fairly correlates with patien vmptom and is an important abnormality which usually cessitates digitization (Fig. 5b). Rectal intussuscept, on is classified into mucosal intussusception or full thickness intussusception (Fig. 3d) (Video 2). This causes obstruction to the passage of feces. MR defecography is advantageous in discriminating between mucosal intussusception and full-thickness intussusception and is relevant in treatment planning. Mucosal intussuscepti n can be treated with transanal excision of the redu dant or prolapsing mucosa, whereas a rectopexy migh e required for full-thickness intussusception [12]. Ente defined as caudal displacement of smal wel loo ps into the recto-vesical or recto-vaginal opace, curs more commonly in patients who hav undergon hysterectomy owing to disruption of $\mathrm{pu}$-cervidal and rectovaginal portions of supportin. ndo fascia. Enteroceles are more clearly d monst. le towards the end of defecation process b ca a full/, loaded rectum does not allow sufficient space i descent of small bowel into pelvis $[12,16,1, \ldots$ is vital 20 detect enterocele because it forms a cor in on for stapled transanal rectal resection (STAR due to the potential danger to the herniated ll bovel during this surgery $[11,18]$. Abnormal caudar descent of bladder and cervix below pubo-coccyseal line is also graded into mild, moderate, an vere (Fig. 5a). Abnormal pelvic floor descent grading c 1 be easily remembered by the rule of 3 with desit of an organ below PCL by $\leq 3 \mathrm{~cm}$ mild descent, 3 $6 \mathrm{~cm}$ moderate descent, and $>6 \mathrm{~cm}$ severe descent $[8$, 12, 13]. Defecation phase puts the maximum downward force on pelvic floor which helps in demonstration of a higher number of pelvic organ descents when compared to strain phase $[15,19]$. Ano-rectal junction descent was

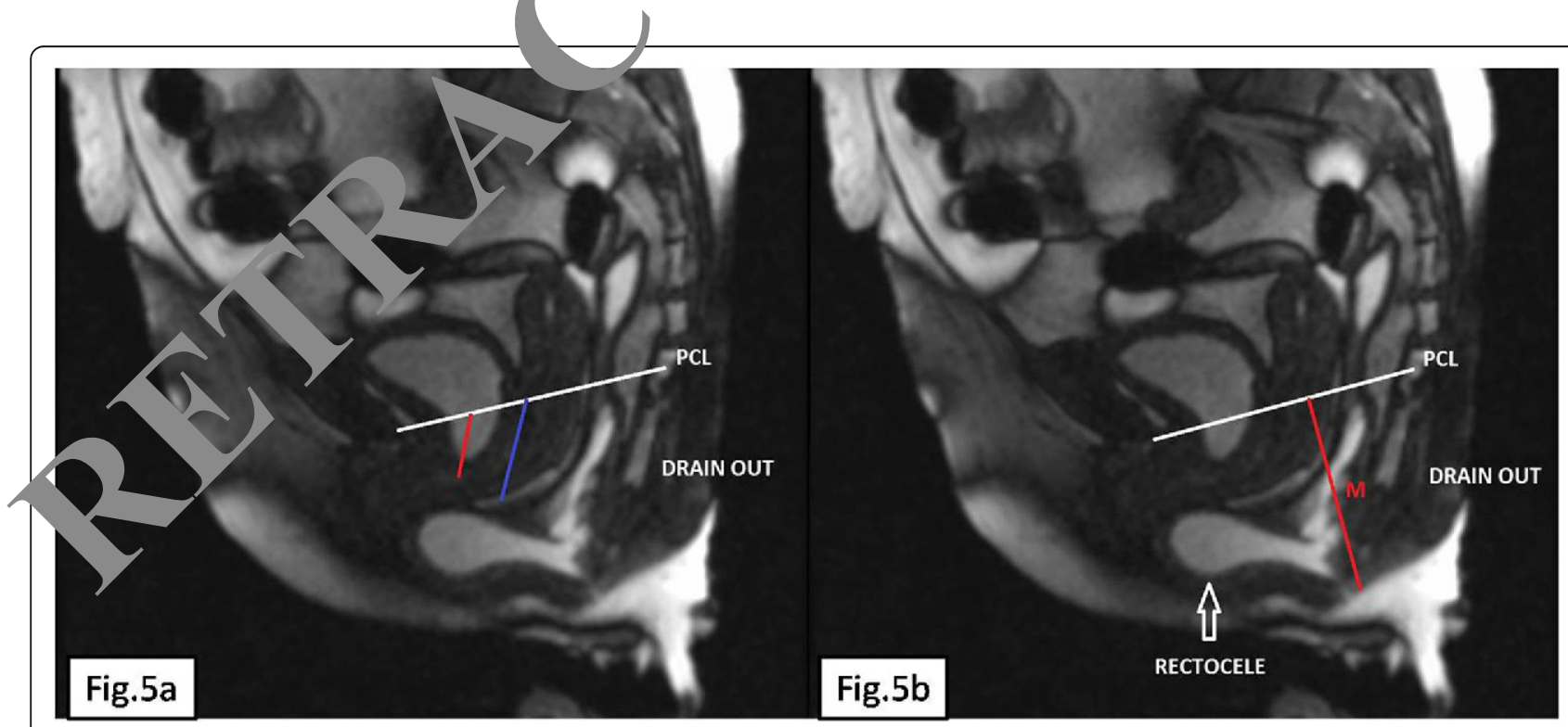

Fig. 5 Descent of all the compartments. Terminal drain out (defecation phase) of same patient as in Fig. 4 reveals descent of bladder neck (red line) and cervix (blue line) (a). Same patient also shows severe ano-rectal junction decent (red line) with retention of jelly in anterior rectocele (white arrow) (b). This picture highlights the role of running the defecation phase imaging for a sufficient time to demonstrate the full abnormality 
visible in 101 (36.9\%) patients on strain phase which increased to 273 in defecation phase. Thus, defecation phase clearly has higher detection rate for ano-rectal junction descent [20]. Similarly, bladder and cervical descent were seen in 16 and 32 patients during strain phase and in 92 and 78 patients respectively during defecation or drain out phase. Defecation phase also identified an additional number 81 (34.9\%) rectoceles when compared to strain phase. None of the patients showed intussusceptions during strain, and all the 93 patients of intussusception were identified during defecation phase. Also, we noted that the maximum depth or degree of an abnormality was visible during defecation phase (Fig. 4c, d). So clearly, the diagnostic yield of defecation phase is best among all the phases of defecation and this attests to the fact that defecation phase is the single most important phase to elicit the full range of pelvic floor abnormalities and must be included in magnetic resonance defecography (Video 3 and Video 4). This comes at a slightly higher cost of providing the patient with waterproof diaper and having to explain the patient to defecate on MRI table which might be little embarrassing to many patients.

\section{Conclusion}

A vast range of pelvic floor abnormalities existing m various combinations in ODS patients can be a strated and graded using MRD which in tur nelp choosing the best treatment plan for 0 patien. Defecation phase is the single most important se of MRD and has the highest diagnostic yield and must be included in all MRD studies.

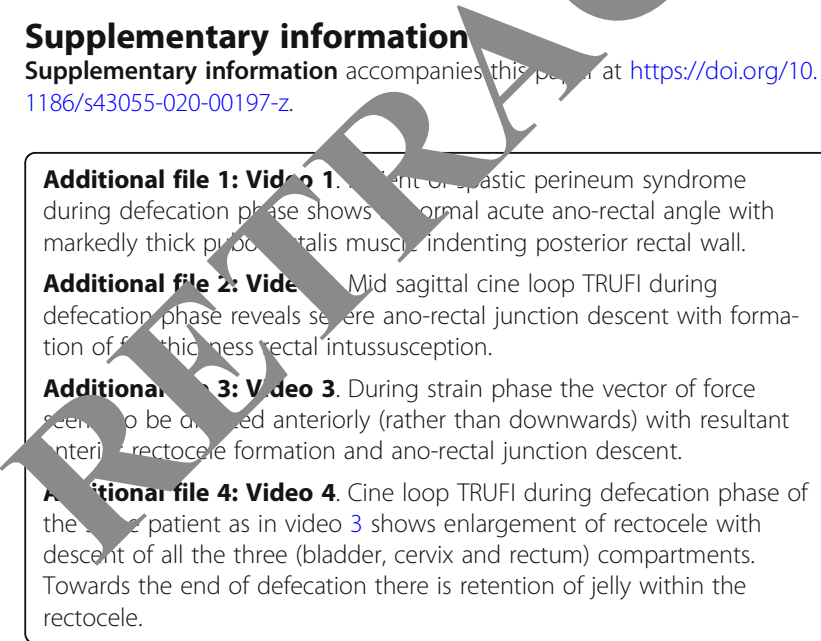

\section{Abbreviations}

MRD: Magnetic resonance defecography; MRI: Magnetic resonance imaging; ODS: Obstructed defecation syndrome; SPS: Spastic perineum syndrome; PCL: Pubo-coccygeal line; H line: Hiatal line; NICE: National Institute for health and Clinical Excellence; TRUFI: True fast imaging with steady state free precession

\section{Acknowledgements}

None.

\section{Authors' contributions}

PA and WA performed, analyzed, and interpreted the magnetic resonance defecography images. Both the authors were involved in manuscript preparation and literature research. Both the authors have read and approved the manuscript.

\section{Funding}

No funding was required for this study as it was the part/of evaluation per the institutional protocol. The patients paid themselve nomina feer for the procedure.

\section{Availability of data and materials} All the data and materials were obtained'from tients re jistered in our hospital.

\section{Ethics approval and consent articipate}

This study was duly approve by nstitutional Ethical Committee (IEC) of Sher-i-Kashmir Institute of Medical Sc os (SKIMS) under the No. SIMS 037/ IEC-SKIMS/2016-45. No an I participa/ts were used in this study. Informed verbal consent war aine

\section{Consent fol nublication}

None

\section{Competing int rests}

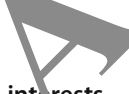

Jare that we have no (financial and non-financial) competing

iv_d: 12 March 2020 Accepted: 5 May 2020

$\mathrm{Pu}$ ished online: 15 May 2020

\section{References}

1. Thapar RB, Patankar RV, Kamat RD, Thapar RR, Chemburkar V (2015 Jan) MR defecography for obstructed defecation syndrome. The Indian journal of radiology \& imaging 25(1):25

2. Longstreth GF, Thompson WG, Chey WD, Houghton LA, Mearin F, Spiller RC (2006) Functional bowel disorders. Gastroenterology. 130(5):1480-1491

3. Mohamed F. O, Ahmed FA. Role of MR Defecography in the assessment of obstructed defecation syndrome. The Medical Journal of Cairo University 2018;86(March):927-931.

4. Bamboriya R, Jaipal U, Jakhar S (2020) A descriptive study of MR defecography for evaluation of obstructed defecation syndrome. International Journal of Medical and Biomedical Studies 31:4(1)

5. Lembo A, Camilleri M (2003) Chronic constipation. N Engl J Med 349:1360-1368

6. Garcia del Salto L, de Miguel CJ, Aguilera del Hoyo LF et al (2014) MR imaging-based assessment of the female pelvic floor. Radiographics. 34(5): 1417-1439

7. Schreyer AG, Paetzel C, Furst A et al (2012) Dynamic magnetic resonance defecography in 10 asymptomatic volunteers. World J Gastroenterol 18(46): 6836-6842

8. Law YM, Fielding JR (2008) MRI of pelvic floor dysfunction: review. AJR Am J Roentgenol 191(6 Suppl):S45-S53

9. Faccioli N, Comai A, Mainardi P, Perandini S, Farah M, Pozzi-Mucelli R (2010) Defecography: a practical approach. Diagn Interv Radiol 16:209-216

10. Roos JE, Weishaupt D, Wildermuth $S$ et al (2002) Experience of 4 years with open MR defecography: pictorial review of anorectal anatomy and disease. Radiographics. 22(4):817-832

11. Pannu HK, Kaufman HS, Cundiff GW et al (2000) Dynamic MR imaging of pelvic organ prolapse: spectrum of abnormalities. Radiographics. 20(6):1567-1582

12. Colaiacomo MC, Masselli G, Polettini E et al (2009) Dynamic MR imaging of the pelvic floor: a pictorial review. Radiographics. 29(3):e35

13. Boyadzhyan L, Raman SS, Raz S (2008) Role of static and dynamic MR imaging in surgical pelvic floor dysfunction. Radiographics. 28(4):949-967

14. Elshazly WG, El Nekady AA, Hassan H (2010) Role of dynamic magnetic resonance imaging in management of obstructed defecation case series. Int J Surg 8:274-282 
15. Liu G, Cui Z, Dai Y, Yao Q, Xu J, Wu G (2017) Paradoxical puborectalis syndrome on diffusion-weighted imaging: a retrospective study of 72 cases. Sci Rep 7(1):1-6

16. Woodfield CA, Hampton BS, Sung V, Brody JM (2009) Magnetic resonance imaging of pelvic organ prolapse: comparing pubococcygeal and midpubic lines with clinical staging. Int Urogynecol J Pelvic Floor Dysfunct 20(6):695-701

17. Alt CD, Brocker KA, Lenz F, Sohn C, Kauczor HU, Hallscheidt P (2014) MRI findings before and after prolapse surgery. Acta Radiol 55(4):495-504

18. McNevin MS (2010) Overview of pelvic floor disorders. Surg Clin N Am 90: 195-205

19. Fielding JR (2002) Practical MR imaging of female pelvic floor weakness. Radiographics. 22(2):295-304

20. DeLancey JO (1994) The anatomy of the pelvic floor. Curr Opin Obstet Gynecol 6(4):313-316

\section{Publisher's Note}

Springer Nature remains neutral with regard to jurisdictional claims in published maps and institutional affiliations.

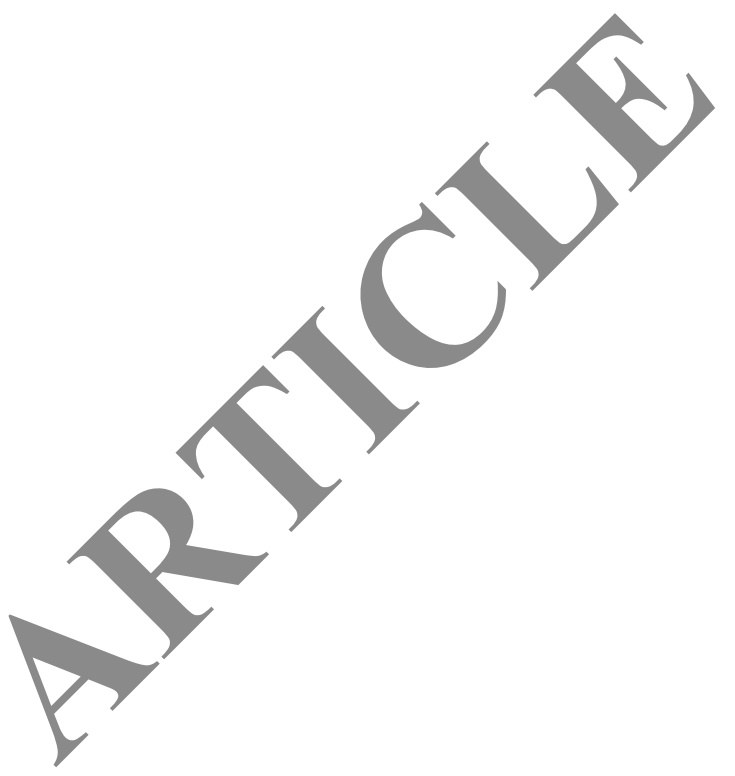

Submit your manuscript to a SpringerOpen ${ }^{\circ}$ journal and benefit from:

- Convenient online submission

- Rigorous peer review

- Open access: articles freely available online

High visibility within the field

- Retaining the copyright to your article 